\title{
Forschungsfelder für Künstliche Intelligenz in der strategischen Produktplanung
}

\author{
Patrick Ködding ${ }^{1(\mathbb{})}$ und Roman Dumitrescu ${ }^{1,2}$ \\ ${ }^{1}$ Heinz Nixdorf Institut, Universität Paderborn, 33102 Paderborn, Deutschland \\ \{Patrick.Koedding, Roman. Dumitrescu\}@hni.upb.de \\ ${ }^{2}$ Fraunhofer Institut für Entwurfstechnik Mechatronik, \\ 33102 Paderborn, Deutschland
}

\begin{abstract}
Zusammenfassung. Der Megatrend Digitalisierung durchdringt alle Bereiche des täglichen Lebens von Unternehmen und Individuen. Insbesondere das produzierende Gewerbe befindet sich in einem tiefgreifenden Wandel. Die Digitalisierung löst als Schlüsseltreiber der Innovationen des 21. Jahrhunderts grundlegende Veränderungen in der Produktentstehung aus. Auf der einen Seite bieten sich zahlreiche Erfolg versprechende Möglichkeiten durch den Einsatz neuer Technologien, eingebetteter Systeme und neuer Ansätze in der Datenverarbeitung. Auf der anderen Seite steigt dadurch aber auch die Komplexität der intelligenten, technischen Systeme. Immer kürzer werdende Entwicklungszyklen, immer größere Datenmengen sowie die steigende Komplexität der neuen Marktleistungen stellen Unternehmen vor große Herausforderungen. Die Entwicklung, Implementierung und Nutzung von Anwendungen der künstlichen Intelligenz (KI) eröffnet Unternehmen die Möglichkeit, nicht nur diese Herausforderungen zu meistern, sondern auch vielfältige Nutzenpotenziale in der Produktentstehung zu erschließen. Dies ist Gegenstand des vorliegenden Beitrags. Zunächst wird daher das Spannungsfeld aus KI und Produktentstehung analysiert. Überdies werden die mit der Einführung, Entwicklung und Nutzung von KI-Anwendungen verbundenen Potenziale und Herausforderungen gezeigt. Abschließend werden auf dieser Basis Forschungsfelder für KI in der strategischen Produktplanung abgeleitet. Aspekte der digitalen Souveränität und verwandte Themen - wie Erklärbarkeit der KI-Anwendungen und Verfügbarkeit interner Kompetenzen - spielen eine zentrale Rolle, wenn es darum geht, Potenziale der KI für die strategische Produktplanung zu nutzen.
\end{abstract}

Schlüsselwörter: Künstliche Intelligenz · Strategische Produktplanung · Digitalisierung · Soziotechnische Betrachtung · Digitale Souveränität · Forschungsfelder

\section{Einführung}

Der Produktentstehungsprozess ist die treibende Kraft hinter Produktinnovationen. Er umfasst alle Aufgaben von der ersten Idee bis hin zur fertigen Marktleistung (Gausemeier et al. 2019). Bei der Entwicklung neuer Marktleistungen werden hier bereits bis zu $85 \%$ der späteren Herstellungskosten festgelegt (Coenenberg 2003). Durch die voranschreitende Digitalisierung nimmt die Bedeutung der Produktentstehung 
weiter zu. Die Digitalisierung durchdringt alle Lebensbereiche und löst tiefgreifende Veränderungen in der Produktentstehung aus (Noll et al. 2016). Im Rahmen der Digitalisierung stehen intelligente technische Systeme im Mittelpunkt der Betrachtung. Hierbei handelt es sich um mechatronische Systeme, die über eine inhärente Teilintelligenz verfügen. Sie besitzen zudem die Fähigkeit, über das Internet mit anderen Systemen zu kommunizieren und zu kooperieren (Dumitrescu und Gausemeier 2018; Kühn 2017). Darüber hinaus gewinnen auch digitale, datenbasierte Services immer mehr an Bedeutung. Sie stellen Dienstleistungen dar, die mit Hilfe von Informationsund Kommunikationstechnologien oftmals über das Internet erbracht werden und eng auf eine Sachleistung abgestimmt sind (Echterfeld et al. 2017; Reichwald und Meier 2002). Gleichzeitig setzt die voranschreitende Digitalisierung Unternehmen unter immer größeren Zeitdruck. Neue, komplexe Marktleistungen in Form von intelligenten technischen Systemen und digitalen Services sowie technologische Neuerungen müssen in immer kürzeren Entwicklungszyklen realisiert werden (Winter 2017; Gausemeier et al. 2013). Folglich steigen die Anforderungen an die Produktentstehung und damit auch an die strategische Produktplanung.

Ein Lösungsansatz sind Anwendungen, die auf Verfahren künstlicher Intelligenz (KI) beruhen. Sie sind dabei, branchenübergreifende Veränderungen herbeizuführen (Buxmann und Schmidt 2019; Wahlmüller-Schiller 2017; acatech 2016). Für Deutschland wird das KI-induzierte zusätzliche Wachstum für die Wirtschaft auf 1,3 bis $2 \%$ geschätzt (McKinsey Global Institute 2018; Purdy und Daugherty 2016). Weltweit wird das Wachstum für KI im produzierenden Gewerbe auf knapp $50 \%$ jährlich bis zu einem Marktvolumen von 17 Mrd. US\$ im Jahr 2025 geschätzt (MarketsandMarkets 2018). Speziell für das produzierende Gewerbe in Deutschland lässt sich bis 2023 eine zusätzliche Bruttowertschöpfung von 2,3\% jährlich - oder kumuliert 32 Mrd. € - erwarten (Seifert et al. 2018). Der Einsatz von KI verspricht vielfältige Potenziale für die Produktentstehung und kann als zentraler Treiber für die Digitalisierung aufgefasst werden (Bitkom 2018; Hecker et al. 2017). In diesem Beitrag werden die sich daraus ergebenden Forschungsfelder für KI in der Produktentstehung im Allgemeinen und der strategischen Produktplanung im Speziellen hergeleitet und beschrieben.

\section{Das Spannungsfeld aus KI und Produktentstehung}

Die Planung und Entwicklung von intelligenten technischen Systemen und Dienstleistungen basiert auf dem Zusammenspiel verschiedener Fachdisziplinen wie der Mechanik, Elektronik und Informationstechnik (Gausemeier et al. 2019). Der Produktentstehungsprozess dieser immer komplexer werdenden Marktleistungen beinhaltet alle Prozessschritte von der Geschäftsidee bis hin zum Serienanlauf (Gausemeier et al. 2019; Feldhusen und Grote 2013). Die strategische Produktplanung ist integraler Bestandteil der Produktentstehung. Insgesamt umfasst die Produktentstehung vier Hauptaufgabenbereiche: Strategische Produktplanung, Produkt-, Dienstleistungs- und Produktionssystementwicklung. Diese Hauptaufgabenbereiche dürfen dabei nicht als stringente Abfolge von Phasen und Meilensteinen verstanden werden. Vielmehr handelt es sich um ein Wechselspiel unterschiedlicher 
Aufgaben (Gausemeier et al. 2019; Gausemeier et al. 2014). Das Referenzmodell der strategischen Planung und integrativen Entwicklung von Marktleistungen, auch 4-Zyklen-Modell der Produktentstehung genannt, strukturiert das integrative und iterative Zusammenwirken der Aufgaben in vier Zyklen (siehe Abb. 1) (Gausemeier et al. 2019; Gausemeier et al. 2014):

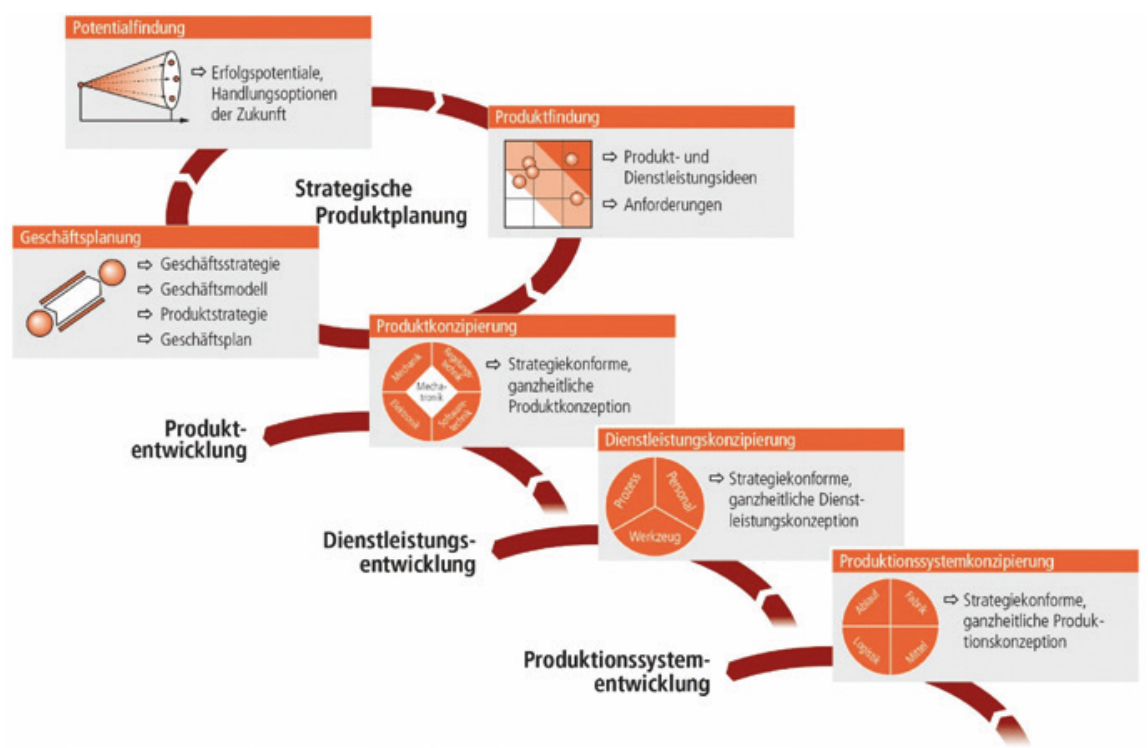

Abb. 1. Referenzmodell der strategischen Planung und integrativen Entwicklung von Marktleistungen nach (Gausemeier et al. 2019; Gausemeier et al. 2014)

\section{Erster Zyklus: Strategische Produktplanung}

Der erste Zyklus dient zur Erarbeitung eines erfolgversprechenden Entwicklungsauftrags. Er umfasst die Phasen Potenzial- und Produktfindung sowie Geschäftsplanung. Im Zuge der Potenzialfindung gilt es, künftige Erfolgspotenziale und darauf aufbauende Handlungsoptionen zu identifizieren. Auf dieser Grundlage werden in der Produktfindung Produkt- und Dienstleistungsideen generiert. Die Geschäftsplanung setzt sich mit der Erstellung einer Geschäftsstrategie und der Ausarbeitung eines Geschäftsmodells und einer Produktstrategie auseinander. Letztere wiederum mündet schließlich in einen Geschäftsplan. Dieser erbringt den Nachweis, ob ein attraktiver Return on Invest (ROI) zu erzielen ist (Gausemeier et al. 2019; Gausemeier et al. 2014; Gausemeier und Plass 2014).

\section{Zweiter Zyklus: Produktentwicklung}

Produktkonzipierung, Entwurf, Ausarbeitung und Produktintegration bilden die Aufgabenbereiche der Produktentwicklung. Schnittstelle zwischen den ersten beiden Zyklen ist die Produktkonzipierung. Ihr Ziel besteht in der Prinziplösung für das beabsichtigte Produkt. Diese enthält unter anderem einen Anforderungskatalog, eine Funktionshierarchie und ein Gestaltmodell. Die Prinziplösung ist der Ausgangspunkt für den domänenspezifischen Entwurf sowie die Ausarbeitung und Integration 
der Ergebnisse aus den Fachdisziplinen Mechanik, Elektronik, Regelungstechnik und Softwaretechnik zu einer verifizierten Gesamtlösung (Gausemeier et al. 2019; Gausemeier et al. 2014).

\section{Dritter Zyklus: Dienstleistungsentwicklung}

Ziel der Dienstleistungsentwicklung ist die Umsetzung einer Dienstleistungsidee in eine Marktleistung (Bullinger et al. 2006). Auch hierbei handelt es sich um keine stringente Abfolge von Phasen und Meilensteinen, sondern um ein Wechselspiel der Aufgaben Dienstleistungskonzipierung, -planung und -integration. Die Dienstleistungskonzipierung umfasst die Aspekte Prozess, Personal und Werkzeug und wird in der Dienstleistungsplanung weiter konkretisiert, bevor die erzielten Ergebnisse dann in der Dienstleistungsintegration vereinigt werden (Gausemeier et al. 2019; Gausemeier et al. 2016; Gausemeier et al. 2014).

\section{Vierter Zyklus: Produktionssystementwicklung}

Ausgangspunkt des vierten Zyklus ist die Konzipierung des Produktionssystems, welche im Zusammenspiel mit der Produktkonzipierung erarbeitet wird. In der Produktionssystementwicklung werden die Fachgebiete Arbeitsmittel-, Arbeitsablaufund Arbeitsstättenplanung sowie Produktionslogistik bearbeitet. Analog zur Produktentwicklung erfolgt die abschließende Integration $\mathrm{zu}$ einem verifizierten Produktionssystem (Gausemeier et al. 2019; Gausemeier et al. 2016).

Produkt-, Dienstleistungs- und Produktionssystementwicklung sind eng aufeinander abgestimmt voranzutreiben. Nur so lässt sich sicherstellen, dass alle Möglichkeiten der Gestaltung einer leistungsfähigen, kostengünstigen und innovativen Marktleistung ausgeschöpft werden (Gausemeier et al. 2019; Gausemeier et al. 2014).

KI-Verfahren bieten die Möglichkeit, mit der steigenden Komplexität der immer softwarelastigeren Marktleistungen und den resultierenden immensen Datenmengen im Produktentstehungsprozess effizient umzugehen (Hecker et al. 2017). Insbesondere für wissensintensive Tätigkeiten wie in der Produktentstehung bietet KI große Nutzenpotenziale (siehe Abb. 2). So können KI-Anwendungen signifikant Entwicklungszeiten reduzieren, Entwicklungskapazitäten erhöhen, Entwicklungsrisiken vermindern und Herstellungskosten senken (Dumitrescu et al. 2020a; Geissbauer et al. 2019; McKinsey \& Company 2017).

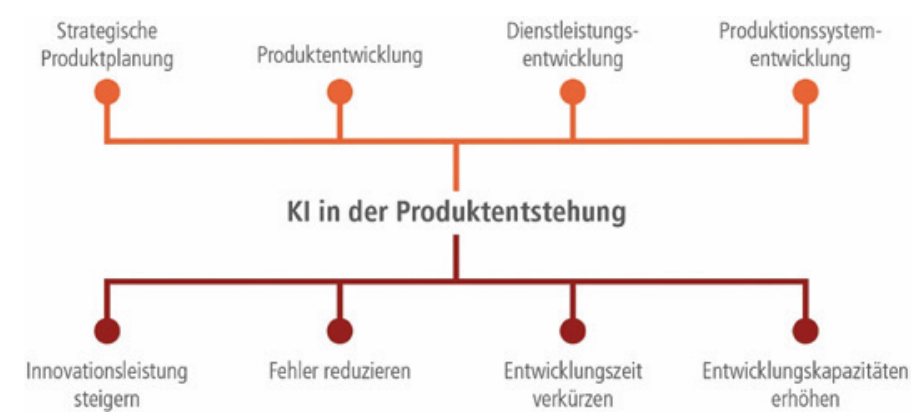

Abb. 2. Allgemeine Nutzenpotenziale von KI in der Produktentstehung in Anlehnung an (Dumitrescu et al. 2020b; Geissbauer et al. 2019; McKinsey \& Company 2017) 
Mit Hilfe von KI-Anwendungen lassen sich Daten verarbeiten und automatisch Schlussfolgerungen ableiten (Satzger et al. 2019). Dabei können Daten entlang des gesamten Lebenszyklus von Marktleistungen gesammelt und analysiert werden, um Wissen für den Produktentstehungsprozess zu generieren (Bretz et al. 2018). Es existieren bereits einige KI-Anwendungen, die die spezifischen Bedarfe der Produktentstehung adressieren. Beispielsweise verwenden Unternehmen aus der Luft- und Raumfahrtindustrie KI-Algorithmen für Generative Design, um Flugzeugteile mit völlig neuen Designs zu entwickeln. Die entsprechenden KI-Algorithmen untersuchen alle möglichen Designlösungen unter Berücksichtigung vorab definierter Ziele und Randbedingungen. Durch iteratives Testen und Lernen werden Designs optimiert und Lösungen vorgeschlagen, die dem menschlichen Verstand unkonventionell erscheinen können (Küpper et al. 2018). Weiterhin wird in der Anforderungsanalyse - Verarbeitung und Analyse natürlicher Sprache eingesetzt, um textuelle Anforderungen zu analysieren und ihre Qualität zu evaluieren (Liu 2018). Aber auch in der strategischen Produktplanung selbst kommen verschiedene KI-Anwendungen zum Einsatz. KI-Anwendungen können Prognosemodelle erstellen, abstimmen und bereitstellen, um z. B. Vorhersagen für die Verbrauchernachfrage zu ermitteln (Anodot 2020). Darüber hinaus können mit Hilfe von Text Mining-Ansätzen große Datenmengen aus wissenschaftlichen Publikationen, Internetquellen, Patenten etc. analysiert und auf ihre Relevanz hin überprüft werden (Minghui et al. 2018). Auf diese Weise lässt sich z. B. Wissen für die Technologiefrühaufklärung oder auch für Trendanalysen generieren (Fraunhofer INT 2020).

Die skizzierten Einsatzmöglichkeiten verdeutlichen das große Nutzenpotenzial von KI-Anwendungen für die Produktentstehung im Allgemeinen sowie die strategische Produktplanung im Speziellen. Bei der Einführung von KI-Anwendungen benötigen viele Unternehmen jedoch Unterstützung. Denn obwohl Deutschland im Bereich der KI-Forschung überdurchschnittlich gut aufgestellt ist, gestaltet sich die Überführung der Forschungsergebnisse in die Industrie schwierig (Seifert et al. 2018). Insbesondere können Unternehmen oft nicht einschätzen, welche Möglichkeiten mit der Nutzung von KI-Anwendungen einhergehen (Dukino et al. 2020). Für einen erfolgreichen Transfer der Ergebnisse aus der Forschung in die Industrie sind daher eine tiefergehende, multiperspektivische Betrachtung der dezidierten Potenziale und Herausforderungen im Kontext der strategischen Produktplanung (siehe Kap. 3) sowie die Ermittlung anwendungsnaher bzw. -integrierter Forschungsfelder unausweichlich (siehe Kap. 4).

\section{Herausforderungen für Unternehmen}

KI-Anwendungen bieten Unternehmen mannigfaltige Nutzenpotenziale (siehe Kap. 2), stellen sie aber gleichzeitig auch vor gänzlich neue Herausforderungen. Diese Potenziale und Herausforderungen lassen sich zeitlich entlang der unternehmerischen Planungsebenen (strategisch, taktisch, operativ) sowie inhaltlich anhand von soziotechnischen Handlungsfeldern (Mensch, Technik, Organisation, Business) strukturieren (Frank et al. 2018). 
Auf strategischer Ebene werden die unternehmerische Vision und die Strategien $\mathrm{zu}$ deren Umsetzung definiert. Ziel ist eine langfristig vorteilhafte Wettbewerbsposition für das Unternehmen. Auf taktischer Ebene werden mittelfristige Entscheidungen getroffen, die sich an den in der Strategie definierten Leitlinien ausrichten sollten. Diese betreffen unter anderem das Wertschöpfungssystem und Geschäftsmodelle. Entscheidungen mit kurzfristigen Erfolgswirkungen werden auf operativer Ebene getroffen. Die operativen Tätigkeiten ergeben sich unmittelbar aus den taktischen Vorgaben und dem Tagesgeschäft (Porter 1996; Adam 1996).

Die Einführung und Nutzung von KI-Anwendungen wirken sich auf verschiedene Ebenen eines Unternehmens aus. Für eine holistische soziotechnische Analyse der Herausforderungen und Potenziale für Unternehmen gilt es, die klassischen Handlungsfelder Mensch, Technik und Organisation um das Handlungsfeld Business zu ergänzen (Frank et al. 2018; Ulich 2011).

Das Handlungsfeld Mensch umfasst alle Aspekte, die die Gestaltung der Arbeit im Kontext von KI-Anwendungen betreffen. Hierzu gehören insbesondere die benötigten Arbeitsinhalte und Kompetenzen (Frank et al. 2018). KI-Technologien bzw. die Technik liefern die Basis für KI-Anwendungen. Diese Technologien befähigen KI-Anwendungen dazu, ihre Umgebung wahrzunehmen und zu verstehen, Informationen $\mathrm{zu}$ verarbeiten, eigenständig Entscheidungen $\mathrm{zu}$ treffen, entsprechend zu handeln sowie kontinuierlich aus deren Folgen zu lernen und mit Menschen und anderen Systemen zu interagieren (Russell und Norvig 1995). Die inner- und zwischenbetrieblichen Prozesse sowie Wertschöpfungsschritte, die für die Einführung, Nutzung aber auch die Entwicklung von KI-Anwendungen vonnöten sind, zählen zur Organisation. Die ergänzenden wirtschaftlichen Aspekte wie zum Beispiel neuartige Geschäftsmodelle werden unter dem Handlungsfeld Business subsumiert (Frank et al. 2018).

Mithilfe einer explorativen Freitextsuche in Google Scholar sowie in Google wurden zahlreiche wissenschaftliche Publikationen und Studien in Bezug auf den Einsatz von KI in Unternehmen, KI in der Produktentstehung bzw. der strategischen Produktplanung sowie $\mathrm{zu}$ den Herausforderungen und Potenzialen für Unternehmen im Kontext von KI-Anwendungen identifiziert. Durch Prüfung auf Titelund Abstract-Ebene konnten zehn Publikationen und Studien für eine detaillierte Betrachtung extrahiert werden. Im Zuge dieser Analyse wurden diese Quellen auf Herausforderungen und Potenziale vor dem Hintergrund der beiden Dimensionen bzw. der soziotechnischen Handlungsfelder und Planungsebenen analysiert. Abb. 3 zeigt eine Sammlung von Herausforderungen und Potenzialen für Unternehmen bei der Einführung und Nutzung von KI-Anwendungen. Diese aufgeführten Beispiele wurden in den wissenschaftlichen Publikationen häufig genannt bzw. ihnen wurde in den untersuchten Studien eine hohe Relevanz für Unternehmen beigemessen. Als besonders erfolgskritisch für Unternehmen haben sich dabei folgende vier Herausforderungen herausgestellt: Die Robustheit und Erklärbarkeit von KI-Anwendungen, die digitale Souveränität sowie die erforderlichen Kompetenzen. 


\begin{tabular}{|c|c|c|c|c|}
\hline & Mensch & Technik & Organisation & Business \\
\hline 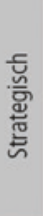 & $\begin{array}{l}4 \text { Gefährdung von } \\
\text { Arbeitsplätzen } \\
4 \text { Ethischer Umgang mit } \\
\text { KI-Anwendungen }\end{array}$ & $\begin{array}{l}\oplus \text { Digitale Souveränität } \\
4 \text { Erklärbarkeit von } \\
\text { KI-Anwendungen }\end{array}$ & $\begin{array}{l}4 \text { Gestaltung der Auf- } \\
\text { bau- und Ablauforga- } \\
\text { nisation } \\
4 \text { Verkürzung von } \\
\text { Bearbeitungs- und } \\
\text { Durchlaufzeiten }\end{array}$ & $\begin{array}{l}4 \text { Differenzierung } \\
\text { gegenüber Wett- } \\
\text { bewerbern } \\
4 \text { Integration in Digitali- } \\
\text { sierungsstrategie }\end{array}$ \\
\hline 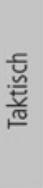 & $\begin{array}{l}\oplus \text { Fokussierung auf } \\
\text { Kerntätigkeiten } \\
4 \text { Gestaltung der } \\
\text { Mensch-Technik- } \\
\text { Interaktion }\end{array}$ & $\begin{array}{l}4 \text { Robustheit und } \\
\text { Effizienz der KI- } \\
\text { Algorithmen } \\
4 \text { Datenschutz und } \\
\text { Datensicherheit }\end{array}$ & $\begin{array}{l}\oplus \text { Verbesserte Entschei- } \\
\text { dungsqualität } \\
4 \text { Hohe Kosten bei } \\
\text { Einführung }\end{array}$ & $\begin{array}{l}\text { ๑ Neue Möglichkeiten } \\
\text { für Geschäftsmodelle } \\
4 \text { Marktmacht von KI- } \\
\text { Anbietern }\end{array}$ \\
\hline 㐫 & $\begin{array}{l}4 \text { Fehlende Kompeten- } \\
\text { zen und Fachkräfte } \\
4 \text { Mitarbeiterakzeptanz }\end{array}$ & $\begin{array}{l}\oplus \text { Effizienzsteigerun- } \\
\text { gen, Prozess- und } \\
\text { Qualitätsoptimierung } \\
4 \text { Datenqualität der } \\
\text { Trainingsdaten }\end{array}$ & $\begin{array}{l}4 \text { Integration von KI- } \\
\text { Anwendungen in be- } \\
\text { stehende Strukturen } \\
4 \text { Mangel an klar defi- } \\
\text { nierten Prozessen }\end{array}$ & $\begin{array}{l}\oplus \text { Besseres Kunden- } \\
\text { verständnis durch } \\
\text { Nutzung von Kl- } \\
\text { Anwendungen } \\
4 \text { Unklarheit über An- } \\
\text { wendung und Nutzen } \\
\text { von KI-Anwendungen }\end{array}$ \\
\hline
\end{tabular}

Abb. 3. Herausforderungen und Potenziale von KI-Anwendungen nach Planungsebene und Handlungsfeld

\section{Robustheit}

Die Robustheit von KI-Algorithmen lässt sich hinsichtlich dreier Aspekte spezifizieren. Erstens dürfen KI-Anwendungen keine Verzerrungen aufweisen. Diese können durch eine fehlende Datenqualität oder eine $\mathrm{zu}$ geringe respektive unausgewogene Datenbasis hervorgerufen werden. Hat ein Unternehmen beispielsweise bislang verstärkt Ingenieurinnen und Ingenieure für die strategische Planung und Entwicklung von Marktleistungen eingesetzt, wird die KI-Anwendung diese Berufsgruppe auch weiterhin empfehlen. Der Grund dafür sind unausgewogene Trainingsdaten und damit verbunden fehlende Erfahrungswerte für weitere Berufsgruppen. Zweitens müssen sich KI-Anwendungen robust gegenüber Eingriffen von außen erweisen. Dies gilt insbesondere auch für externe Einflüsse, die im regulären Betrieb auf die Lernkomponente einer KI-Anwendung einwirken. Ein Beispiel für die fehlende Robustheit ist der Kommunikationsbot Tay von Microsoft, der nach wenigen Stunden aufgrund von erlerntem rassistischen Verhalten bereits wieder abgeschaltet worden ist. Drittens müssen KI-Anwendungen zuverlässige Ergebnisse der Datenanalyse für Ausreißer und in unerwarteten Situationen liefern. Für autonome Fahrzeuge ist es beispielsweise unabdingbar, partiell verdeckte Objekte einwandfrei klassifizieren und entsprechende Entscheidungen ableiten zu können (Satzger et al. 2019; Lundborg und Märkel 2019; Seifert et al. 2018; Bitkom 2018; Sickert 2016). 


\section{Erklärbarkeit}

Die Verständlichkeit und Nachvollziehbarkeit der von KI-Anwendungen bereitgestellten Unterstützung bzw. der getroffenen Entscheidungen stellen wichtige Kriterien für die Akzeptanz von und das Vertrauen für diese Lösungen dar. Es reicht hierbei nicht aus, wenn das Ergebnis begründet werden kann. Vielmehr müssen auch das Zustandekommen sowie die hierfür erforderlichen Schlussfolgerungen transparent erläutert werden. Eine Möglichkeit zur Erhöhung der Transparenz kann durch die Bereitstellung von kontextspezifischen Informationen oder die Verwendung von interpretierbaren Algorithmen geleistet werden. Allerdings sind leistungsfähige Algorithmen häufig aufgrund ihrer Komplexität nur unzureichend erklärbar. Es resultiert ein Konflikt zwischen der Qualität der Ergebnisse und der Erklärbarkeit der Algorithmen. Weiterhin müssen nicht nur einzelne Ergebnisse, sondern auch die Entwicklung und Adaption von KI-Anwendungen kontrollierbar sein. Hierfür gilt es, die zugrunde liegenden Daten und genutzten Verfahren zur Entwicklung von KI-Anwendungen sowie jegliche Änderungen im Zeitverlauf lückenlos zu dokumentieren (Satzger et al. 2019; Lundborg und Märkel 2019; Bitkom 2018). Weitere Aspekte der Erklärbarkeit und insbesondere auch Hinweise auf Methoden für ihre Herstellung finden sich im Beitrag von Florian Eiling und Marco Huber (in diesem Band).

\section{Digitale Souveränität}

Die Erklärbarkeit der KI-Anwendungen ist bereits eine wesentliche Voraussetzung für die digitale Souveränität, hinsichtlich der Individuen wie auch hinsichtlich der Unternehmen. Weiterhin müssen die Nutzung, der Zugang und die Verwertung von Daten eindeutig geregelt sein, um einen souveränen Umgang mit den Daten für KI-Anwendungen für Beschäftigte, Gruppen und Unternehmen gewährleisten zu können. Alle Akteure müssen eigenständig darüber entscheiden und transparent einsehen können, welche Daten für welche Zwecke freigegeben sind und verwendet werden. Diese Anforderung gewinnt durch die exponentiell ansteigenden, verfügbaren und auswertbaren Datenmengen immer mehr an Bedeutung (Satzger et al. 2019; Seifert et al. 2018; Hecker et al. 2017). Übergreifend lässt sich digitale Souveränität als die Möglichkeit und Fähigkeit auffassen, digitale Technologien in diesem Kontext KI-Anwendungen - zielgerichtet und kompetent für die eigenen Zwecke so einzusetzen, dass die eigene Handlungsfähigkeit und Kompetenz der einzelnen Akteure gestärkt wird (Institut für Innovation und Technik 2019). Folglich müssen nicht nur technische Voraussetzungen für den souveränen Umgang mit Daten geschaffen werden, sondern auch ausreichend Kompetenzen und Fachkräfte für die effiziente Nutzung von KI-Anwendungen verfügbar sein.

\section{Kompetenzen}

Eine weitere zentrale Herausforderung bei der Entwicklung und Einführung von KI-Anwendungen stellen fehlende interne Kompetenzen und Fachkräfte dar. Insbesondere besteht in Unternehmen ein hoher Bedarf an Data Scientists, aber auch an erfahrenen Projektmanagern und KI-Forschern. Dies spiegelt sich unter anderem auch darin wider, dass viele Unternehmen nicht einschätzen können, was mit KI-Anwendungen heutzutage bereits möglich ist (Deloitte 2020; Dukino et al. 2020; 
Geretshuber und Reese 2019; Lundborg und Märkel 2019; Seifert et al. 2018; Hecker et al. 2017).

Abschließend lässt sich konstatieren, dass die Entwicklung, Implementierung und Nutzung von KI-Anwendungen vielfältige Nutzenpotenziale für Unternehmen in der strategischen Produktplanung bieten. Gleichzeitig sehen sich Unternehmen mit zahlreichen Herausforderungen in den Dimensionen Mensch, Technik, Organisation und Business konfrontiert, wie zum Beispiel bei der Robustheit, der Erklärbarkeit, der digitalen Souveränität und den erforderlichen Kompetenzen.

\section{Forschungsfelder}

Aus einer Analyse der beschriebenen Potenziale und Herausforderungen lassen sich mehrere Forschungsfelder für KI in der strategischen Produktplanung ableiten, die diese Herausforderungen adressieren und den Transfer der Erkenntnisse in Unternehmen unterstützen. Insgesamt ergeben sich fünf aufeinander aufbauende Forschungsfelder: Potenzialfindung im Produktentstehungsprozess, Strukturierung von KI-Anwendungen, Mensch-Technik-Interaktion, Spezifikationstechnik für KI-Anwendungen und Kompetenzaufbau. Nachfolgend werden die einzelnen Forschungsfelder hergeleitet und beschrieben.

\section{Potenzialfindung im Produktentstehungsprozess}

Unternehmen stehen bei der Einführung, Entwicklung und Nutzung von KI-Anwendungen zunächst vor der Herausforderung, die Einsatzmöglichkeiten und den damit verbundenen Nutzen von KI-Anwendungen für das eigene Geschäft $\mathrm{zu}$ ermitteln und $\mathrm{zu}$ bewerten. Folglich ist eine Charakterisierung des Produktentstehungsprozesses mit besonderem Fokus auf die strategische Produktplanung erforderlich, um feststellen zu können, ob und wie KI-Anwendungen Prozesse und Tätigkeiten der Produktentstehung unterstützen können. Die holistische Betrachtungsweise wird benötigt, da die strategische Produktplanung eng mit der Produkt-, Dienstleistungs- und Produktionssystementwicklung verzahnt ist (siehe Kap. 2). Gleichzeitig müssen auch die zugehörigen Datenquellen, Daten und IT-Systeme sowie ihre Abhängigkeiten untereinander aufgenommen und strukturiert werden. Auf dieser Basis können Potenziale für KI-Anwendungen innerhalb der strategischen Produktplanung abgeleitet werden. Die Bewertung und Auswahl von adäquaten KI-Anwendungen zur Erschließung der identifizierten Potenziale erfordern jedoch einen Überblick über bestehende KI-Anwendungen im Kontext der strategischen Produktplanung.

\section{Strukturierung von KI-Anwendungen}

Eine strukturierte Übersicht über KI-Anwendungen erhöht das Verständnis für KI-Anwendungen und kann einen Beitrag zur Bewertung der Robustheit und Erklärbarkeit liefern. Erstens bedarf es dafür einer Identifikation und Dokumentation existierender KI-Anwendungen im Produktentstehungsprozess bzw. der strategischen Produktplanung. Zweitens müssen die ermittelten KI-Anwendungen anhand geeigneter Kriterien, wie zum Beispiel den verwendeten KI-Technologien, -Verfahren 
und Methoden, strukturiert und klassifiziert werden. Eine Möglichkeit zur Generierung einer zweidimensionalen Anwendungsübersicht bietet die multidimensionale Skalierung. Die Anwendungsübersicht unterstützt damit die Auswahl von KIAnwendungen. Nachgelagerte Schritte wie die Einführung oder Entwicklung werden nur indirekt adressiert. Insbesondere muss hierbei die Symbiose zwischen KI und Mensch in der strategischen Produktplanung gestaltet werden.

\section{Mensch-Technik-Interaktion}

KI-Anwendungen verändern die Interaktion zwischen Mensch und Maschine bzw. Technik. Zur Steigerung der Akzeptanz muss die Mensch-Technik-Interaktion digital souverän gestaltet werden. Die Beschreibung und Analyse der Mensch-Technik-Interaktion beim Einsatz von konventionellen sowie digitalen Technologien bilden den Ausgangspunkt für die Analyse der Besonderheiten bei der Mensch-Technik-Interaktion im Kontext von KI-Anwendungen. Hieraus lassen sich wichtige Implikationen für die benutzungsfreundliche Gestaltung der veränderten Mensch-Technik-Interaktion ableiten; ein wesentlicher Aspekt ist dabei die Erklärbarkeit von KI-Anwendungen aus der Perspektive der Nutzenden. Bei der Gestaltung der Mensch-Technik-Interaktion sowie der eigentlichen Entwicklung von KI-Anwendungen müssen Unternehmen darüber hinaus domänenübergreifend durch eine Spezifikationstechnik unterstützt werden.

\section{Spezifikationstechnik für KI-Anwendungen}

Durch eine Spezifikationstechnik lässt sich die Nachvollziehbarkeit von KI-Anwendungen steigern, sowohl bei der Entwicklung als auch im Betrieb. Bislang existiert keine solche Spezifikationstechnik. Die Spezifikationstechnik muss während der Planung und Konzipierung sowie Veränderungen im Betrieb bei der Erarbeitung, Dokumentation und Beschreibung der erzielten Ergebnisse unterstützen, um die Robustheit und Erklärbarkeit von KI-Anwendungen zu steigern. Hierfür müssen grafische Notationselemente und Regeln zu deren Verwendung bereitgestellt werden, die u. a. die Besonderheiten der Mensch-Technik-Interaktion widerspiegeln. Dies kann entweder durch die Weiterentwicklung bestehender oder die Entwicklung einer neuen Spezifikationstechnik für KI-Anwendungen geschehen. Um ein umfassendes Verständnis trivialer und komplexer Sachverhalte auch ohne große Einarbeitungszeit zu gewährleisten, ist eine semiformale Spezifikationstechnik anzustreben (Schneider 2018; Gausemeier et al. 2001; Chouikha et al. 1998). Überdies sehen sich Unternehmen mit einem übergreifenden Querschnittsthema in der strategischen Produktplanung konfrontiert: neuartigen Kompetenzanforderungen.

\section{Kompetenzaufbau}

Für die Entwicklung, Implementierung und Nutzung von KI-Anwendungen benötigen Mitarbeiter und Unternehmen andere Kompetenzen als für bisherige Marktleistungen. Erstens gilt es, den veränderten Kompetenzbedarf für die erfolgreiche Benutzung der KI-Anwendungen in Unternehmen zu ermitteln. Zweitens müssen Kompetenzcluster, zum Beispiel in Form von Kompetenzrollen, identifiziert werden, die unter Erschließung von Synergieeffekten gemeinsam aufgebaut werden können. Drittens folgt die Ableitung von prototypischen Aufbaumaßnahmen für den identifizierten Kompetenzbedarf. 
Die fünf aufeinander aufbauenden, übergeordneten Forschungsfelder adressieren allgemein die verschiedenen Herausforderungen (siehe Kap. 3) im Kontext von KI-Anwendungen in der strategischen Produktplanung. Diese Forschungsfelder lassen sich spezifisch sehr unterschiedlich ausprägen. Für das erste Forschungsfeld, Potenzialfindung in der Produktentstehung, lassen sich sowohl direkte als auch indirekte Potenziale ableiten. Direkte Potenziale beziehen sich auf die Einführung und Nutzung von KI-Anwendungen für die Tätigkeiten innerhalb der strategischen Produktplanung. Indirekte Potenziale ergeben sich hingegen aus den neuen Aufgaben, die mit der Einführung und Nutzung von KI verbunden sind. Nachfolgend werden exemplarisch je ein direktes und indirektes Potenzial inklusive zugrundeliegender Forschungsfragen vorgestellt. Ein Beispiel für ein direktes Potenzial in der strategischen Produktplanung ist die KI-gestützte Vorausschau, ein Beispiel für ein indirektes Potenzial die Entwicklung von Produktstrategien für datenbasierte Services:

\section{KI-gestützte Vorausschau}

Im Gegensatz zur Potenzialfindung im Produktentstehungsprozess geht es bei der KI-gestützten Vorausschau nicht um die Identifikation von Potenzialen für KI-Anwendungen, sondern um die Anwendung von KI, um Erfolgspotenziale im Rahmen der Vorausschau zu ermitteln. KI-Anwendungen können bestehende Methoden, wie z. B. die Szenario-Technik, unterstützen oder für deren gezielte Weiterentwicklung eingesetzt werden. Insbesondere die Fähigkeit von KI, Massendaten $\mathrm{zu}$ erfassen, $\mathrm{zu}$ analysieren und daraus Schlüsse $\mathrm{zu}$ ziehen, verspricht ein hohes Nutzenpotenzial (acatech 2020). Gleichzeitig kann die benutzungsfreundliche Gestaltung der Mensch-Technik-Interaktion die Anwendungsfreundlichkeit von Methoden der Vorausschau erhöhen und damit den Nutzungsgrad vor allem in kleinen und mittleren Unternehmen steigern.

\section{Produktstrategien für datenbasierte Services}

Bei digitalen, datenbasierten Services, wie zum Beispiel auf KI-Anwendungen basierenden Services, handelt es sich um eigenständige Marktleistungen. Genau wie physische Produkte müssen auch datenbasierte Services strategisch geplant werden. Sie unterscheiden sich aber durch ihre spezifischen Eigenschaften wie die digitale, softwarebasierte Updatefähigkeit von physischen Produkten. Ansätze zur Entwicklung von Produktstrategien für physische Produkte lassen sich folglich nicht ohne Weiteres auf datenbasierte Services übertragen. Insbesondere stehen Unternehmen im Kontext von KI vor der Herausforderung, Möglichkeiten zur langfristigen Differenzierung im Wettbewerb sowie zum Erhalt des Wettbewerbsvorsprungs zu identifizieren (Deloitte 2020; Echterfeld et al. 2017).

\section{Zusammenfassung}

Im Zuge der voranschreitenden Digitalisierung sehen sich viele Unternehmen im Produktentstehungsprozess mit zahlreichen Herausforderungen konfrontiert: Von immer kürzer werdenden Entwicklungszyklen über exponentiell wachsende Datenmengen bis hin $\mathrm{zu}$ hochkomplexen intelligenten, technischen Systemen. 
KI-Anwendungen sind in der Lage, mit den steigenden Anforderungen an die Produktentstehung umzugehen. Vor allem für wissensintensive Tätigkeiten bietet KI große Nutzenpotenziale. Entwicklungsrisiken und -zeiten lassen sich signifikant reduzieren, -kapazitäten erhöhen und Herstellungskosten senken.

Im vorliegenden Beitrag wurden die Potenziale und Herausforderungen bei der Einführung von KI-Anwendungen im Rahmen der strategischen Produktplanung beleuchtet. Es zeigt sich, dass sowohl in den soziotechnischen Dimensionen Mensch, Technik, Organisation und Business als auch in der operativen, taktischen und strategischen Planungsebene zahlreiche Herausforderungen für Unternehmen bestehen. Hierzu zählen insbesondere die Robustheit und Erklärbarkeit von KI-Algorithmen, die digitale Souveränität sowie fehlende Kompetenzen. Aus diesen Herausforderungen lassen sich fünf übergeordnete, aufeinander aufbauende Forschungsfelder für KI in der strategischen Produktplanung identifizieren: Potenzialfindung im Produktentstehungsprozess, Strukturierung von KI-Anwendungen, Mensch-Technik-Interaktion, Spezifikationstechnik für KI-Anwendungen und Kompetenzaufbau. Durch die spezifische Ausprägung dieser Forschungsfelder ergeben sich weitere konkrete Forschungsthemen wie die KI-gestützte Vorausschau oder die Entwicklung von Produktstrategien für datenbasierte Services.

\section{Literatur}

acatech: Innovationspotenziale der Mensch-Maschine-Interaktion. Utz, München (2016)

acatech (Hrsg.): Künstliche Intelligenz in der Industrie. acatech HORIZONTE, München (2020)

Adam, D.: Planung und Entscheidung. Modelle - Ziele - Methoden Mit Fallstudien und Lösungen. Betriebswirtschaftlicher Verlag Gabler, Wiesbaden (1996)

Anodot (Hrsg.): Anodot autonomous forecast (2020). https://www.anodot.com/autonomousforecast/. Zugegriffen: 10. Juli 2020

Bitkom (Hrsg.): Digitalisierung gestalten mit dem Periodensystem der Künstlichen Intelligenz. Ein Navigationssystem für Entscheider. Bundesverband Informationswirtschaft, Telekommunikation und neue Medien e. V., Berlin (2018)

Bretz, L., Foullois, M., Hillebrand, M.: Engineering Intelligence. KI-Kompetenz wird für Entwickler immer wichtiger (2018). https://www.it-production.com/produktentwicklung/kikompetenz-entwickler/. Zugegriffen:15. Juni 2020

Bullinger, H.-J., Scheer, A.-W., Schneider, K.: Service Engineering. Entwicklung und Gestaltung innovativer Dienstleistungen: Mit 24 Tabellen, 2. Aufl. Springer, Berlin (2006)

Buxmann, P., Schmidt, H.: Künstliche Intelligenz. Springer, Berlin (2019)

Chouikha, M., Janhsen, A., Schnieder, E.: Klassifikation und Bewertung von Beschreibungsmitteln für die Automatisierungstechnik. At - Automatisierungstechnik 46(12) (1998). https://doi.org/10.1524/auto.1998.46.12.582

Coenenberg, A.G.: Kostenrechnung und Kostenanalyse - Aufgaben und Lösungen. Hg. von Christian Fink, 3. Aufl. Schäffer-Poeschel, Stuttgart (2003)

Deloitte (Hrsg.): State of AI in the Enterprise - 3rd Edition. Ergebnisse der Befragung von 200 AI-Experten zu Künstlicher Intelligenz in deutschen Unternehmen (2020). https://www2. deloitte.com/content/dam/Deloitte/de/Documents/technology-media-telecommunications/ DELO-6418_State\%20of\%20AI\%202020_KS4.pdf. Zugegriffen: 20. Juli 2020 
Dukino, C., Friedrich, M., Ganz, W., Hämmerle, M., Kötter, F., Meiren, T., Neuhüttler, J, Renner, T., Schuler, S., Zaiser, H.: Künstliche Intelligenz in der Unternehmenspraxis. Studie zu Auswirkungen auf Dienstleistung und Produktion. Hg. von Wilhelm Bauer/Walter Ganz/ Moritz Hämmerle et al. Fraunhofer, Stuttgart (2020)

Dumitrescu, R., Gausemeier, J.: Innovationen im Zeitalter der Digitalisierung. Industrie 4.0 Management 2 (2018)

Dumitrescu, R., Drewel, M., Falkowski, T.: KI-Marktplatz: Das Ökosystem für Künstliche Intelligenz in der Produktentstehung. ZWF 115(1-2), 86-90 (2020a). https://doi. org/10.3139/104.112240

Dumitrescu, R., Foullois, M., Bernijazov, R., Özcan, L., Ködding, P.: Künstliche Intelligenz in der Produktentstehung (im Druck) (2020b)

Echterfeld, J., Dülme, C., Gausemeier, J.: Gestaltung von Produktstrategien im Zeitalter der Digitalisierung. In: Bodden, E., Dressler, F., Dumitrescu, R. (Hrsg.) Wissenschaftsforum Intelligente Technische Systeme (WInTeSys) 2017. 11. und 12. Mai 2017 Heinz Nixdorf MuseumsForum, Paderborn, S. 67-91. Heinz Nixdorf Institut Universität Paderborn, Paderborn (2017)

Feldhusen, J., Grote, K.-H.: Der Produktentstehungsprozess (PEP). In: Feldhusen, J., Grote, K.-H. (Hrsg.) Pahl/Beitz Konstruktionslehre. Methoden und Anwendung erfolgreicher Produktentwicklung, 8. Aufl., S. 11-24. Springer, Berlin (2013)

Frank, M., Koldewey, C., Rabe, M., Dumitrescu, R., Gausemeier, J., Kühn, A.: Smart Services - Konzept einer neuen Marktleistung. ZWF 113(5), 306-311 (2018). https://doi. org/10.3139/104.111913

Fraunhofer INT (Hrsg.): KATI - Knowledge Analytics for Technology \& Innovation (2020). https://www.int.fraunhofer.de/de/geschaeftsfelder/technologie--und-planungsmonitoring/ themen-und-projekte/Kati.html. Zugegriffen: 20. Juli. 2020

Gausemeier, J., Plass, C.: Zukunftsorientierte Unternehmensgestaltung. Strategien, Geschäftsprozesse und IT-Systeme für die Produktion von morgen, 2. Aufl. Hanser, München (2014)

Gausemeier, J., Ebbesmeyer, P., Kallmeyer, F.: Produktinnovation. Strategische Planung und Entwicklung der Produkte von morgen. Hanser, München (2001)

Gausemeier, J., Czaja, A., Wiederkehr, O., Dumitrescu, R., Tschirner, C., Steffen, D.: Studie Systems Engineering in der industriellen Praxis. In: Maurer, M., Schulze, S.-O. (Hrsg.) Tag des Systems Engineering, S. 113-122. Hanser, München (2013)

Gausemeier, J., Amshoff, B., Dülme, C., Kage, M.: Strategische Planung von Marktleistungen im Kontext Industrie 4.0. In: Gausemeier, J. (Hrsg.) Vorausschau und Technologieplanung, S. 6-36. Verlagsschriftenreihe des Heinz Nixdorf Instituts, Paderborn (2014)

Gausemeier, J., Ovtcharova, J., Amshoff, B., Eckelt, D., Elstermann, M., Placzek, M., Wiederkehr, O.: Strategische Produktplanung: adaptierbare Methoden. Prozesse und IT-Werkzeuge für die Planung der Marktleistungen von morgen (2016). https://doi.org/10.2314/GBV:870185012

Gausemeier, J., Dumitrescu, R., Echterfeld, J., Pfänder, T., Steffen, D., Thielemann, F.: Innovationen für die Märkte von morgen. Strategische Planung von Produkten, Dienstleistungen und Geschäftsmodellen. Hanser, München (2019)

Geissbauer, R., Wunderlin, J., Schrauf, S., Krause, J.H., Morr, J.-T., Odenkirchen, A.: Industrie 4.0: Digitale Produktentwicklung verschafft Industrieunternehmen klare Wettbewerbsvorteile (2019). https://www.pwc.de/de/pressemitteilungen/2019/industrie-4-0-digitaleproduktentwicklung-verschafft-industrieunternehmen-klare-wettbewerbsvorteile.html. Zugegriffen: 15. Juli 2020

Geretshuber, D., Reese, H.: Künstliche Intelligenz in Unternehmen. Eine Befragung von 500 Entscheidern deutscher Unternehmen zum Status quo - mit Bewertungen und Handlungsoptionen von PwC (2019). https://www.pwc.de/de/digitale-transformation/kuenstlicheintelligenz/studie-kuenstliche-intelligenz-in-unternehmen.pdf. Zugegriffen: 20. Juli 2020 
Hecker, D., Döbel, I., Rüping, S., Schmitz, V.: Künstliche Intelligenz und die Potenziale des maschinellen Lernens für die Industrie. Wirtschaftsinf. Manag. 9(5), 26-35 (2017). https:// doi.org/10.1007/s35764-017-0110-6

Institut für Innovation und Technik (Hrsg.): Bekanntmachung. Promotionsbegleitende Zuschüsse (2019-2023) im Rahmen eines interdisziplinären Graduiertennetzwerks zum Themenbereich „Maschinenbau der Zukunft“ (2019). https://www.iit-berlin.de/de/aktuelles/ BekanntmachungGraduiertennetzwerkDigitaleSouvernittinderWirtscha...pdf. Zugegriffen: 12. Okt. 2019

Kühn, A.T.: Systematik zur Release-Planung intelligenter technischer Systeme. Universität Paderborn, Paderborn (2017). https://doi.org/10.17619/UNIPB/1-78

Küpper, D., Lorenz, M., Kuhlmann, C., Bouffault, O., van Wyck, J., Köcher, S., Schlageter, J., Lim, Y.H.: AI in the factory of the future (2018). https://www.bcg.com/publications/2018/ artificial-intelligence-factory-future.aspx. Zugegriffen: 25. Juni 2020

Liu, S.: Man plus machine: IBM bringing AI to requirements management (2018). https://www. $\mathrm{ibm}$.com/blogs/internet-of-things/iot-ibm-announces-ai-for-requirements-management/. Zugegriffen: 20. Juli 2020

Lundborg, M., Märkel, C.: Künstliche Intelligenz im Mittelstand. Relevanz, Anwendungen, Transfer. Eine Erhebung der Mittelstand-Digital Begleitforschung (2019). https://www. mittelstand-digital.de/MD/Redaktion/DE/Publikationen/kuenstliche-intelligenz-immittelstand.pdf?_blob=publicationFile \&v=5. Zugegriffen: 20. Juli 2020

MarketsandMarkets (Hrsg.): Artificial intelligence in manufacturing market size, artificial intelligence in manufacturing market by offering (hardware, software, and services), technology (machine learning, computer vision, context-aware computing, and NLP), application, industry, and geography - global forecast to 2025 (2018). https://www.marketsandmarkets.com/Market-Reports/artificial-intelligence-manufacturing-market-72679105.html. Zugegriffen: 10. Juni 2020

McKinsey \& Company (Hrsg.): Smartening up with artificial intelligence (AI). What's in it for Germany and its industrial sector (2017). https://www.mckinsey.de/ /media/McKinsey/ Locations/Europe\%. Zugegriffen: 15. Juli 2020

McKinsey Global Institute (Hrsg.): Notes from the frontier. Modeling the impact of AI on the worldeconomy (2018). https://www.mckinsey.de/ /media/McKinsey/Locations/Europe\%. Zugegriffen: 10. Juni 2020

Minghui, Z., Lingling, Z., Libin, Z., Feng, W.: Research on technology foresight method based on intelligent convergence in open network environment. In: Shi, Y., Fu, H., Tian, Y. (Hrsg.) Computational Science - ICCS 2018, S. 737-747. Springer International Publishing, Cham (2018)

Noll, E., Zisler, K., Neuburger, R., Eberspächer, J., Dowling, M.J. (Hrsg.): Neue Produkte in der digitalen Welt. Norderstedt, Books on Demand (2016)

Porter, M.E.: What is strategy? Harv. Bus. Rev. 74(6), 61-78 (1996)

Purdy, M., Daugherty, P.: Why artificial intelligence is the future of growth (2016). https:// www.accenture.com/t20170524t055435_w__/ca-en/_acnmedia/pdf-52/accenture-why-aiis-the-future-of-growth.pdf. Zugegriffen: 15. Juni 2020

Reichwald, R., Meier, R.: Generierung von Kundenwert mit mobilen Diensten. In: Reichwald, R. (Hrsg.) Mobile Kommunikation, S. 207-230. Gabler, Wiesbaden (2002)

Russell, S.J., Norvig, P.: Artificial intelligence. A modern approach. Prentice Hall, Upper Saddle River (1995)

Satzger, G., Kühl, N., Martin, A.: Unterstützung der Wissensarbeit durch Künstliche Intelligenz - Anforderungen an die Gestaltung maschinellen Lernens. In: Frühjahrstagung 2019 der Gesellschaft für Arbeitswissenschaft GfA, Dortmund (2019) 
Schneider, M.: Spezifikationstechnik zur Beschreibung und Analyse von Wertschöpfungssystemen. Dissertation, Universität Paderborn, HNI-Verlagsschriftenreihe, Bd. 386, Paderborn (2018). https://doi.org/10.17619/UNIPB/1-643

Seifert, I., Bürger, M., Wangler, L., Christmann-Budian, S., Rohde, M., Gabriel, P., Zinke, G.: Potenziale der künstlichen Intelligenz im produzierenden Gewerbe in Deutschland. Institut für Innovation und Technik (iit), Berlin (2018)

Sickert, T.: Künstliche Intelligenz. Vom Hipster-Mädchen zum Hitler-Bot (2016). https:// www.spiegel.de/netzwelt/web/microsoft-twitter-bot-tay-vom-hipstermaedchen-zumhitlerbot-a-1084038.html. Zugegriffen: 20. Juli 2020

Ulich, E.: Arbeitspsychologie, 7. Aufl. Vdf Hochschulverlag AG an der ETH Zürich \& Schöffer-Poeschel, Zürich \& Stuttgart (2011)

Wahlmüller-Schiller, C.: Künstliche Intelligenz - wohin geht die Reise? Elektrotech. Informationstech. 134(7), 361-363 (2017). https://doi.org/10.1007/s00502-017-0529-8

Winter, J.: Europa und die Plattformökonomie - Wie datengetriebene Geschäftsmodelle Wertschöpfungsketten verändern. In: Bruhn, M., Hadwich, K. (Hrsg.) Dienstleistungen 4.0, S. 71-88. Springer Fachmedien Wiesbaden, Wiesbaden (2017)

Open Access Dieses Kapitel wird unter der Creative Commons Namensnennung 4.0 International Lizenz (http://creativecommons.org/licenses/by/4.0/deed.de) veröffentlicht, welche die Nutzung, Vervielfältigung, Bearbeitung, Verbreitung und Wiedergabe in jeglichem Medium und Format erlaubt, sofern Sie den/die ursprünglichen Autor(en) und die Quelle ordnungsgemäß nennen, einen Link zur Creative Commons Lizenz beifügen und angeben, ob Änderungen vorgenommen wurden.

Die in diesem Kapitel enthaltenen Bilder und sonstiges Drittmaterial unterliegen ebenfalls der genannten Creative Commons Lizenz, sofern sich aus der Abbildungslegende nichts anderes ergibt. Sofern das betreffende Material nicht unter der genannten Creative Commons Lizenz steht und die betreffende Handlung nicht nach gesetzlichen Vorschriften erlaubt ist, ist für die oben aufgeführten Weiterverwendungen des Materials die Einwilligung des jeweiligen Rechteinhabers einzuholen.

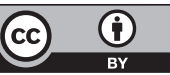

